\title{
Temporary occipital fixation in young children with severe cervical-thoracic spinal deformity
}

\author{
Brian J. Kelley, MD, PhD, ${ }^{1,2}$ Anas A. Minkara, BHS, ${ }^{2}$ Peter D. Angevine, MD, MPH, ${ }^{1}$ \\ Michael G. Vitale, MD, MPH, ${ }^{2}$ Lawrence G. Lenke, MD, ${ }^{2}$ and Richard C. E. Anderson, MD1 \\ 'Department of Neurological Surgery, Neurological Institute; and 'Department of Orthopedic Surgery, Columbia University \\ Medical Center, NewYork-Presbyterian Morgan Stanley Children's Hospital, New York, New York
}

\begin{abstract}
OBJECTIVE The long-term effects of instrumentation and fusion of the occipital-cervical-thoracic spine on spinal growth in young children are poorly understood. To mitigate the effects of this surgery on the growing pediatric spine, the authors report a novel technique used in 4 children with severe cervical-thoracic instability. These patients underwent instrumentation from the occiput to the upper thoracic region for stabilization, but without bone graft at the craniovertebral junction (CVJ). Subsequent surgery was then performed to remove the occipital instrumentation, thereby allowing further growth and increased motion across the CVJ.
\end{abstract}

METHODS Three very young children (15, 30, and 30 months old) underwent occipital to thoracic posterior segmental instrumentation due to cervical or upper thoracic dislocation, progressive kyphosis, and myelopathy. The fourth child (10 years old) underwent similar instrumentation for progressive cervical-thoracic scoliosis. Bone graft was placed at and distal to C-2 only. After follow-up CT scans demonstrated posterior arthrodesis without unintended fusion from the occiput to C-2, 3 patients underwent removal of the occipital instrumentation.

RESULTS Follow-up cervical spine flexion/extension radiographs demonstrated partial restoration of motion at the CVJ. One patient has not had the occipital instrumentation removed yet, because only 4 months have elapsed since her operation.

CONCLUSIONS Temporary fixation to the occiput provides increased biomechanical stability for spinal stabilization in young children, without permanently eliminating motion and growth at the CVJ. This technique can be considered in children who require longer instrumentation constructs for temporary stabilization, but who only need fusion in more limited areas where spinal instability exists.

https://thejns.org/doi/abs/10.3171/2017.7.FOCUS17287

KEY WORDS spinal deformity; occipital fixation; pediatric

$\mathrm{T}$ HE complications of posterior segmental instrumentation and fusion of the thoracic spine in young children are well known, and include impaired pulmonary and cardiac function as well as a reduction in spinal growth and motion. ${ }^{2}$ Although the long-term effects of instrumentation and fusion of the occipital-cervical-thoracic spine in young children are largely unknown, it is generally accepted that performing this surgery prior to skeletal maturity limits spinal growth and mobility. Therefore, in most cases surgical intervention is delayed as long as possible to allow further spinal growth and maturity.

In some situations, however, progressive spinal deformity or spinal cord compression with neurological deterioration develops and surgical intervention is required. In addition to spinal cord decompression as well as reduction and stabilization of the deformity to provide overall spinal balance, another goal of surgery is to preserve skeletal growth and spinal motion by limiting the extent of fusion.

ABBREVIATIONS AP = anteroposterior; $C V J=$ craniovertebral junction; $M A G E C=$ magnetic expansion control; VATER = vertebral defects, anal atresia, tracheoesophageal fistula with esophageal atresia, and radial and renal anomalies; VEPTR = vertical expandable prosthetic titanium rib.

SUBMITTED May 12, 2017. ACCEPTED July 11, 2017.

INCLUDE WHEN CITING DOI: 10.3171/2017.7.FOCUS17287. 
In the thoracolumbar spine, several "growth-friendly" surgical strategies have been developed to achieve deformity correction while preserving spinal growth (e.g., traditional growing rod constructs, vertical expandable prosthetic titanium rib [VEPTR], and magnetically manipulated growing rods-i.e., magnetic expansion control [MAGEC]). ${ }^{9}$ These interventions are designed to control and improve spinal deformity while preserving growth potential within the construct. Although these techniques have been successful in addressing thoracolumbar deformity, comparable strategies have not been developed for the pediatric cervical spine.

When surgery is required in young children with cervical-thoracic deformities, congenital anomalies and the small bones of their anatomy often preclude placement of lateral mass screws or other rigid instrumentation. Therefore, multilevel constructs extending beyond the levels of instability are often necessary to provide additional points of fixation to increase stability, reduce fusion failure, and preclude the need for postoperative bracing. Despite the absence of instability at the craniovertebral junction (CVJ), fixation is often extended to the occiput due to the biomechanical strength of midline occipital screws. ${ }^{4}$ Unfortunately, however, instrumentation to the occiput may reduce spinal growth, and it eliminates motion at the CVJ. 1,8

In an attempt to minimize the effects of occipital-cervical-thoracic fusion on the growing pediatric spine, we report on a novel technique. This technique was used in 4 children with cervical-thoracic pathology who underwent instrumentation from the occiput to the thoracic region for stabilization, but without bone graft at the CVJ. Subsequent surgery was then performed to remove the occipital instrumentation, thereby allowing further growth and increased motion across the CVJ.

\section{Surgical Technique \\ Stage I}

After induction of general anesthesia and placement of electrodes for intraoperative spinal cord monitoring, baseline neurophysiological recordings with the patient in the supine position are obtained. The child is then carefully turned to the prone position, maintaining a neutral neck position. The head is then secured using either a Mayfield head clamp or halo ring with traction. Neurophysiological recordings are repeated and adjustments in positioning are performed if there are any changes. After prepping and draping of the surgical field, a midline incision is made from the occiput to the upper thoracic region. Subperiosteal dissection is performed, exposing bony landmarks for posterior segmental instrumentation from the occiput to the lowest instrumented level (typically T-2 or T-3). Care is taken to avoid violating the joints at the $\mathrm{Oc}-\mathrm{C} 1$ and $\mathrm{C} 1-2$ levels. Upper thoracic pedicle screws, C-2 pars or translaminar screws, and an occipital plate with midline screws in the keel (e.g., 3.5- and 4.0-mm diameter, with variable lengths based on preoperative CT evaluation) are placed. Subaxial lateral mass screws are placed if possible. A temporary rod is placed for stabilization, and decompression is performed if required. Reduction is performed, inter- body support is provided if needed, and final rods (3.5- to $5.5-\mathrm{mm}$ transition) are placed. Final radiographs are obtained. The bony posterior elements are decorticated and local autograft and allograft is placed from C-2 to the thoracic region only. The wound is closed in layers and sterile dressings are applied.

\section{Stage II}

After a subsequent CT scan is performed at approximately 1 year after the initial surgery to confirm solid posterior arthrodesis from $\mathrm{C}-2$ to the thoracic region without unintended fusion across the CVJ, the patient is taken back to the operating room for removal of instrumentation from the occiput to $\mathrm{C}-2$. The operating room setup, patient positioning, and exposure are performed in similar fashion to Stage I. The rods as well as the occipital plate and screws are removed. New rods are placed bilaterally from $\mathrm{C}-2$ to the thoracic region. Wound closure is performed as described above.

\section{Case Reports Case 1}

A 3-month-old boy with vertebral defects, anal atresia, tracheoesophageal fistula with esophageal atresia, and radial and renal anomalies (VATER) syndrome and Sprengel deformity presented with a complete C5-6 spinal dislocation, cervical kyphosis, and multiple congenital vertebral anomalies (Fig. 1A-C). His initial physical examination demonstrated limited range of motion of the neck but spontaneous movements of all extremities, with normal tone and reflexes. Due to his young age and normal examination findings, he was placed in a rigid cervical collar. At 15 months of age he developed myelopathy with bilateral ankle clonus. Repeat upright cervical spine radiographs, CT, and MRI demonstrated progression of the kyphosis and spinal cord compression (Fig. 1D-F).

He was placed in halo traction, with moderate improvement in the deformity compared with baseline (Fig. 2A). In an attempt to achieve greater reduction with traction and avoid an anterior procedure, the patient was taken to the operating room for laminectomy, resection of the dysplastic posterior elements, and partial facetectomies at the level of the dislocation. Additional traction led to further improvement in the kyphosis (Fig. 2B). He then returned to the operating room for posterior spinal instrumentation from the occiput to T-3. Autologous rib graft was harvested and placed from C-2 to T-3, and then secured with cables. Bone morphogenetic protein (extra-small dose) was then overlaid along the fusion construct (Fig. 2C). He was kept in a halo vest for 2.5 months postoperatively.

The patient was lost to follow-up for approximately 5 years due to the family's relocation out of state. After returning to the area and reestablishing care, cervical spine radiographs and $\mathrm{CT}$ scans were performed, demonstrating solid posterior fusion from C-2 to T-3, without unintended fusion from the occiput to C-2. He was taken back to the operating room for removal of the occipital instrumentation. At the time of surgery, no autofusion was observed at the CVJ. At his 9-month follow-up visit, he was able to walk and run with normal strength and sensation in all 

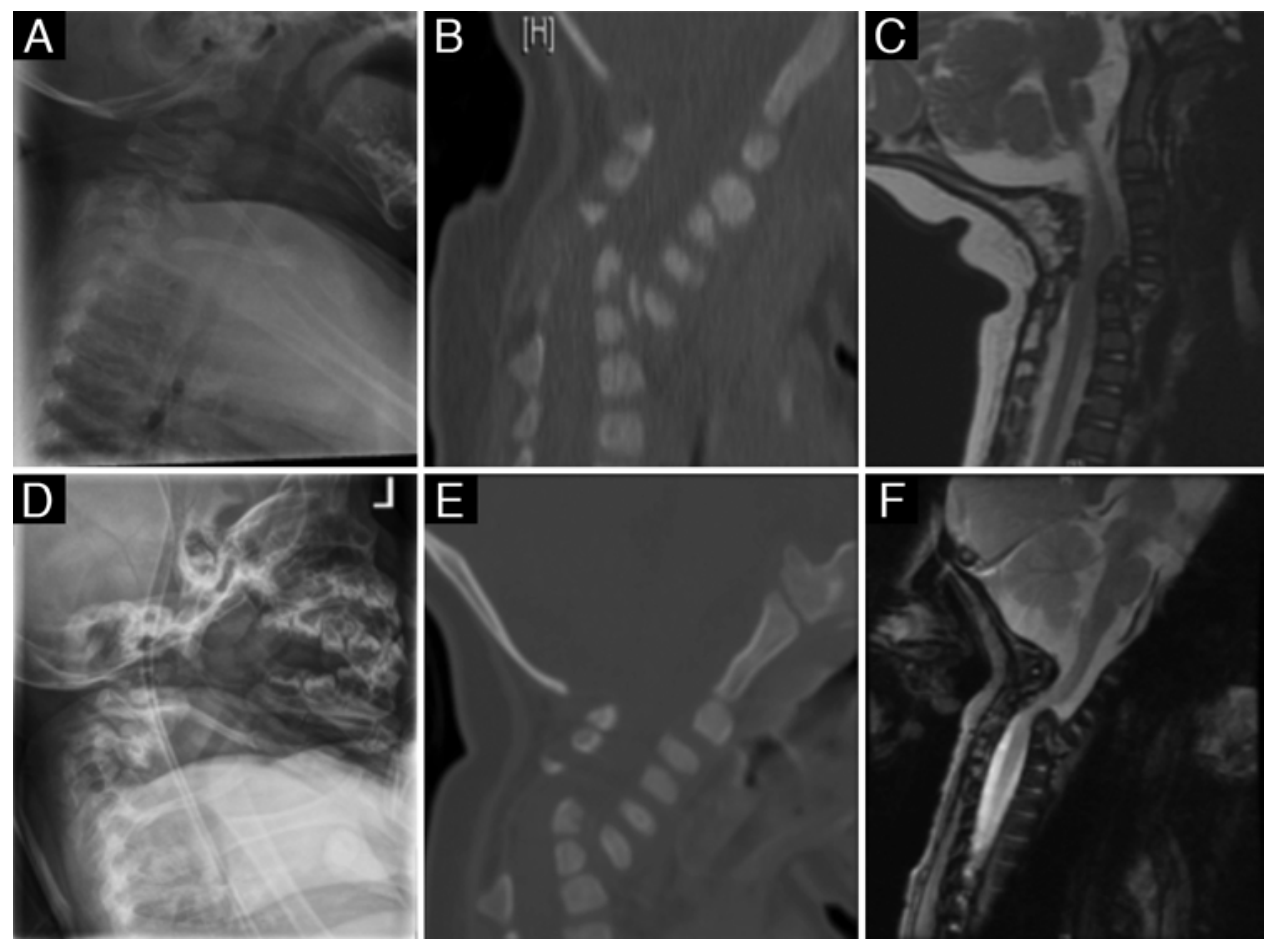

FIG. 1. Case 1. Upright lateral radiographic (A), CT (B), and noncontrast T2-weighted MR (C) images obtained at 3 months of age demonstrate complete C5-6 dislocation with associated canal stenosis. At 15 months of age, repeat lateral radiographic (D), CT $(E)$, and MR (F) images indicate progressive kyphosis with worsening canal stenosis and concern for increased T2-weighted cord signal change within the region of the dislocation site.

extremities and normal reflexes. Cervical spine lateral and flexion/extension radiographs demonstrated limited but improved motion at the CVJ (Fig. 3A-C).

\section{Case 2}

A 30-month-old girl presented with a progressive congenital kyphosis of the cervical-thoracic junction. Initially diagnosed on prenatal ultrasound, her condition was followed clinically from birth until 16 months of age, at which time she was initially treated by halo placement for cervical traction at an outside center. She remained in trac- tion for approximately 9 months until her halo apparatus was removed due to loosening pin sites. On examination, she had normal arm function bilaterally but significant lower-extremity myelopathy, with profound weakness and sustained clonus bilaterally. Upright radiographs, CT, and MRI revealed an acute kyphosis at C7-T1 with severe compression and edema of the spinal cord, along with multiple congenital vertebral anomalies from C-7 to T-2 (Fig. $4 \mathrm{~A}-\mathrm{C})$. Spinal cord tethering was also observed, with the conus terminating at L3-4 and a fatty filum.

She initially underwent lumbar laminectomy for teth-
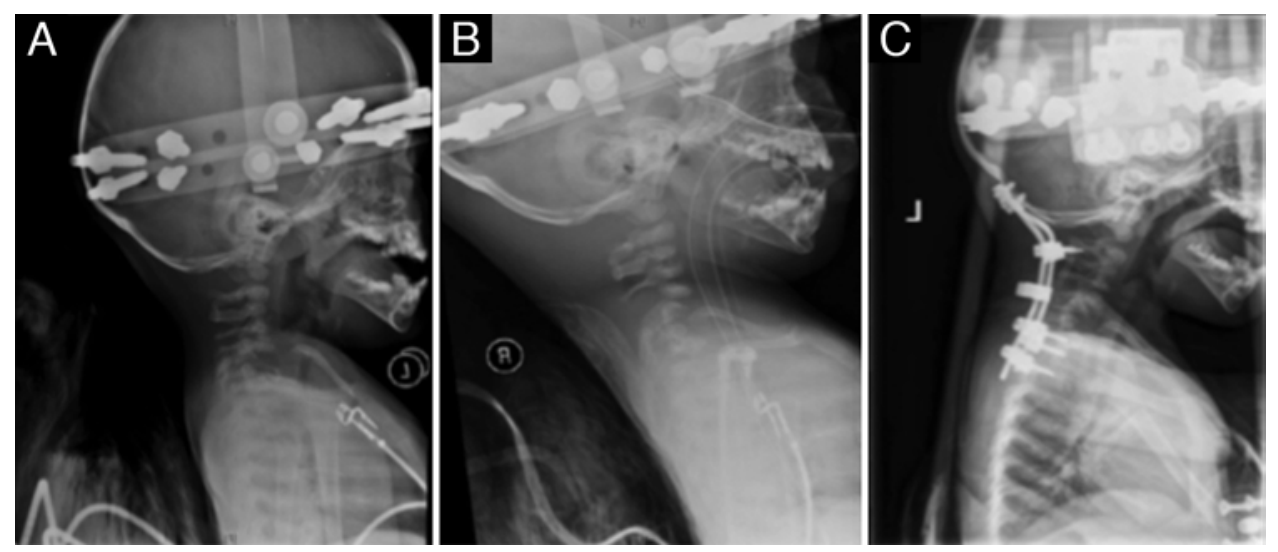

FIG. 2. Case 1. A: Lateral cervical spine radiograph obtained after 4 days of traction indicates some improvement in spinal alignment. B: Lateral radiograph obtained after posterior laminectomy and facetectomies and additional traction shows further improvement in spinal alignment. C: Postoperative lateral radiograph obtained after occipital-cervical-thoracic instrumentation shows improved cervical alignment. 

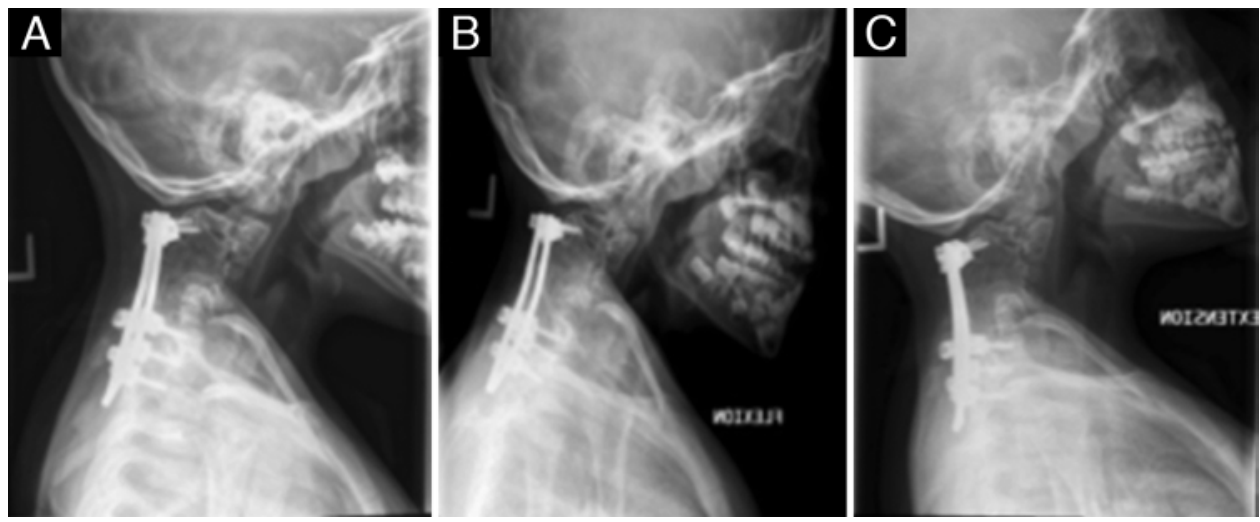

FIG. 3. Case 1. Lateral neutral (A), flexion (B), and extension (C) cervical spine radiographs obtained at 9 months after disconnection of the occipital instrumentation demonstrate improved motion at the CVJ.

ered cord release and halo placement. After 1 week of traction, there was a moderate reduction of the kyphosis compared with baseline. She then underwent posterior segmental instrumentation from the occiput to T-4, vertebral column resection at $\mathrm{T} 1$, and fusion from $\mathrm{C}-2$ to $\mathrm{T}-4$ using rib autograft and allograft bone. On postoperative Day 3, she developed difficulty swallowing. Cervical spine CT demonstrated anterior migration of the interbody graft. Therefore, she returned to the operating room for C-7 and T-2 corpectomies with an interbody allograft strut, and instrumentation from C-6 to T-3 performed using an anterior approach. Anterior instrumentation was placed from C-6 to T-3 using a mandibular fracture plating system, because no traditional anterior spinal plating system was small enough. She was placed in a rigid cervical collar for 3 months.

Three months postoperatively, she was walking with ankle-foot orthotics and minimal support. Her leg strength was almost normal, except dorsiflexion and plantar flexion, which remained at $2 / 5$ strength. Upright radiographs and MRI demonstrated excellent spinal alignment and implant position, with complete decompression of the spinal cord (Fig. 5A-C). At a 6-month follow-up visit, the patient was able to perform unsteady but independent ambulation, including stair climbing. Lower-extremity strength had improved although residual weakness remained. Results on repeat imaging were comparable to findings observed at her 3-month visit. She will continue with physical therapy, and a CT scan is planned for 1 year postoperatively, with subsequent removal of the occipital instrumentation.

\section{Case 3}

A 30-month-old boy presented with significant lowerextremity myelopathy. Imaging demonstrated a congenital dislocation of the upper thoracic spine (Fig. 6). He initially underwent an anterior spinal decompression with anterior and posterior spinal fusion via a posterior approach. Halo vest immobilization was used postoperatively. Three and a half years later he developed a pseudarthrosis with progressive deformity and recurrent myelopathy. He subsequently underwent a vertebral column resection at T-3 and T-4 for circumferential spinal cord decompression, with posterior segmental instrumentation from the occiput to T-10 (Fig. 7A and B). Bone graft was placed posteriorly except at the CVJ. Halo vest immobilization was used
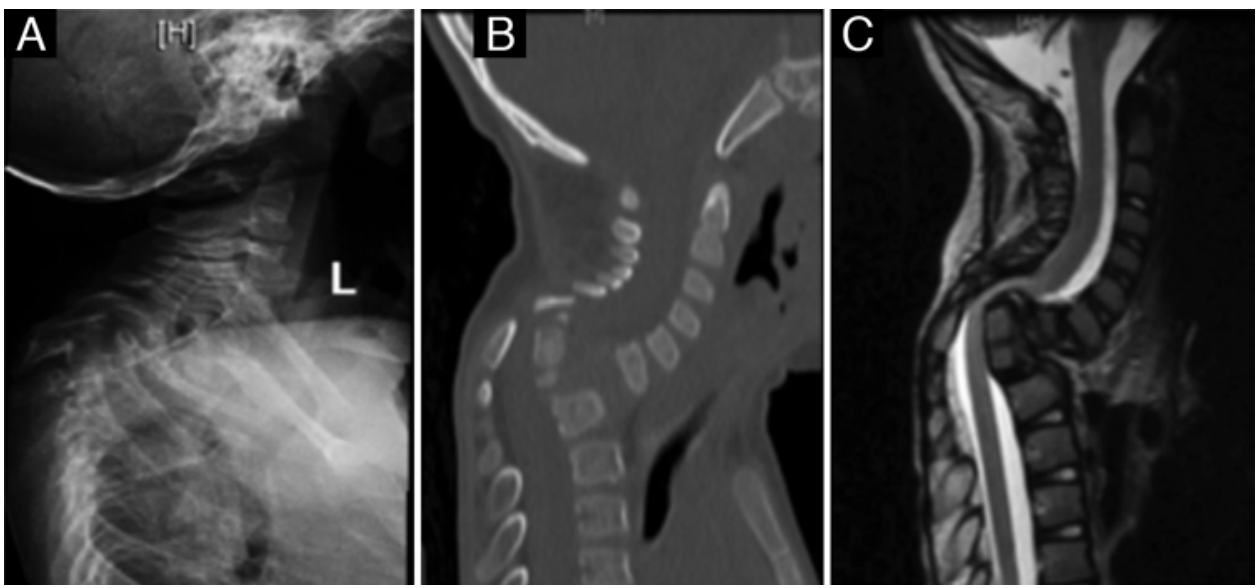

FIG. 4. Case 2. Upright lateral cervical spine radiographic (A), CT (B), and noncontrast T2-weighted MR (C) images of a 30-month-old girl reveal a severe kyphotic deformity at C7-T1 with hypoplastic vertebral bodies and cord compression with edema. 

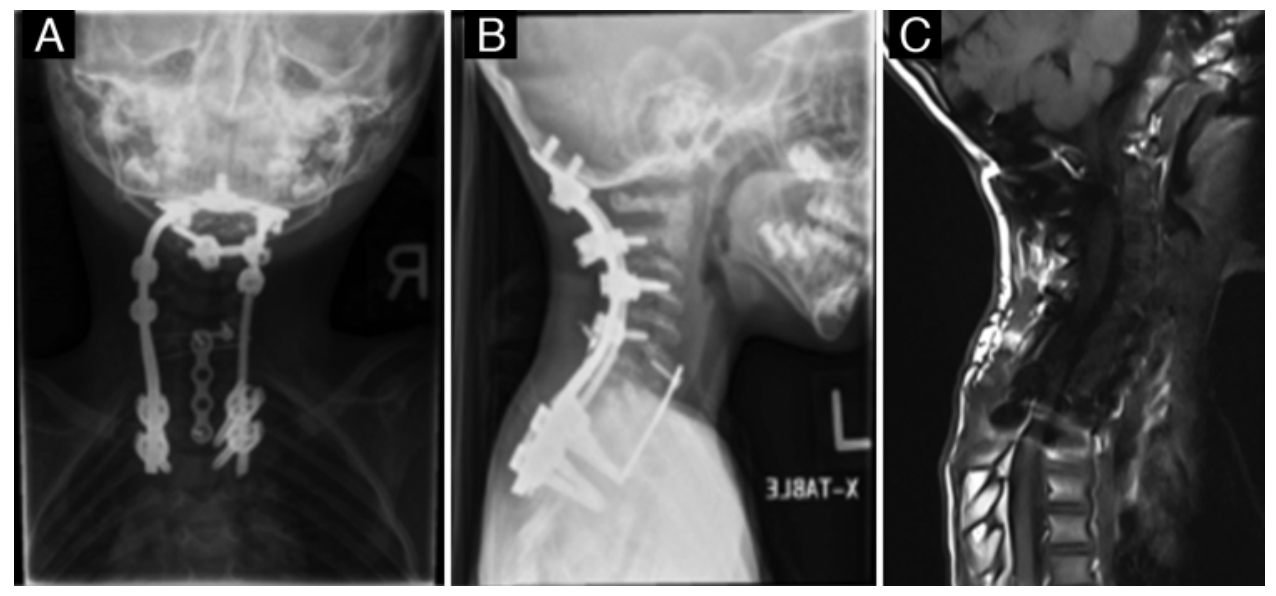

FIG. 5. Case 2. Three months after occipital-cervical-thoracic instrumentation and limited fusion, anteroposterior (AP) (A) and lateral (B) cervical spine radiographs, along with noncontrast T1-weighted MRI (C), indicate restoration of normal cervical alignment and decompression of the cord.

postoperatively. One year later, he returned to the operating room for removal of the occipital instrumentation (Fig. 7C and D).

\section{Case 4}

A 10-year-old boy presented with a severe, progressive neck tilt. Imaging demonstrated a cervical-thoracic congenital scoliosis. He underwent posterior segmental instrumentation from the occiput to T-6, multiple cervical and thoracic posterior column osteotomies, and posterior fusion from C-2 to T-6. Halo vest immobilization was used postoperatively. He returned to the operating room 1 year later for removal of the occipital instrumentation.

\section{Discussion}

In this report, we describe a novel surgical technique for young children with cervical-thoracic spinal deformity in which temporary instrumentation to the occiput without bone graft at the CVJ is used. This provides additional stability to facilitate arthrodesis in the remaining construct, while providing the opportunity to remove the occipital instrumentation at a later time and restore potential growth and motion at the CVJ.

Pediatric cervical-thoracic spinal deformity correction presents unique challenges. The relatively small bone anatomy, potential congenital variations, growth potential, and immature ossification centers often limit conventional
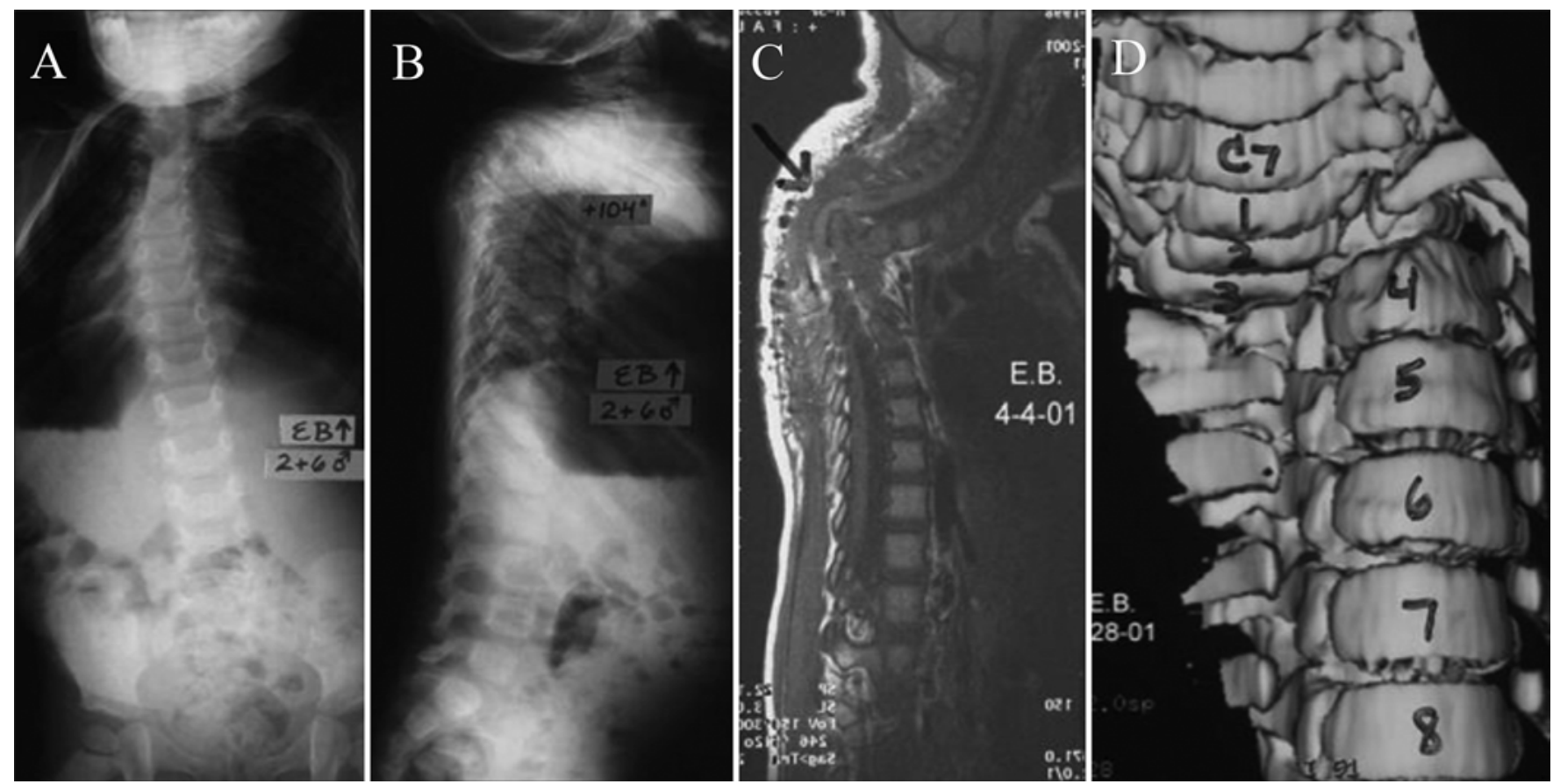

FIG. 6. Case 3. Upright AP (A) and lateral (B) radiographs, T1-weighted MRI (C), and 3D CT reconstruction (D) demonstrate an upper thoracic spinal dislocation and spinal cord compression. 

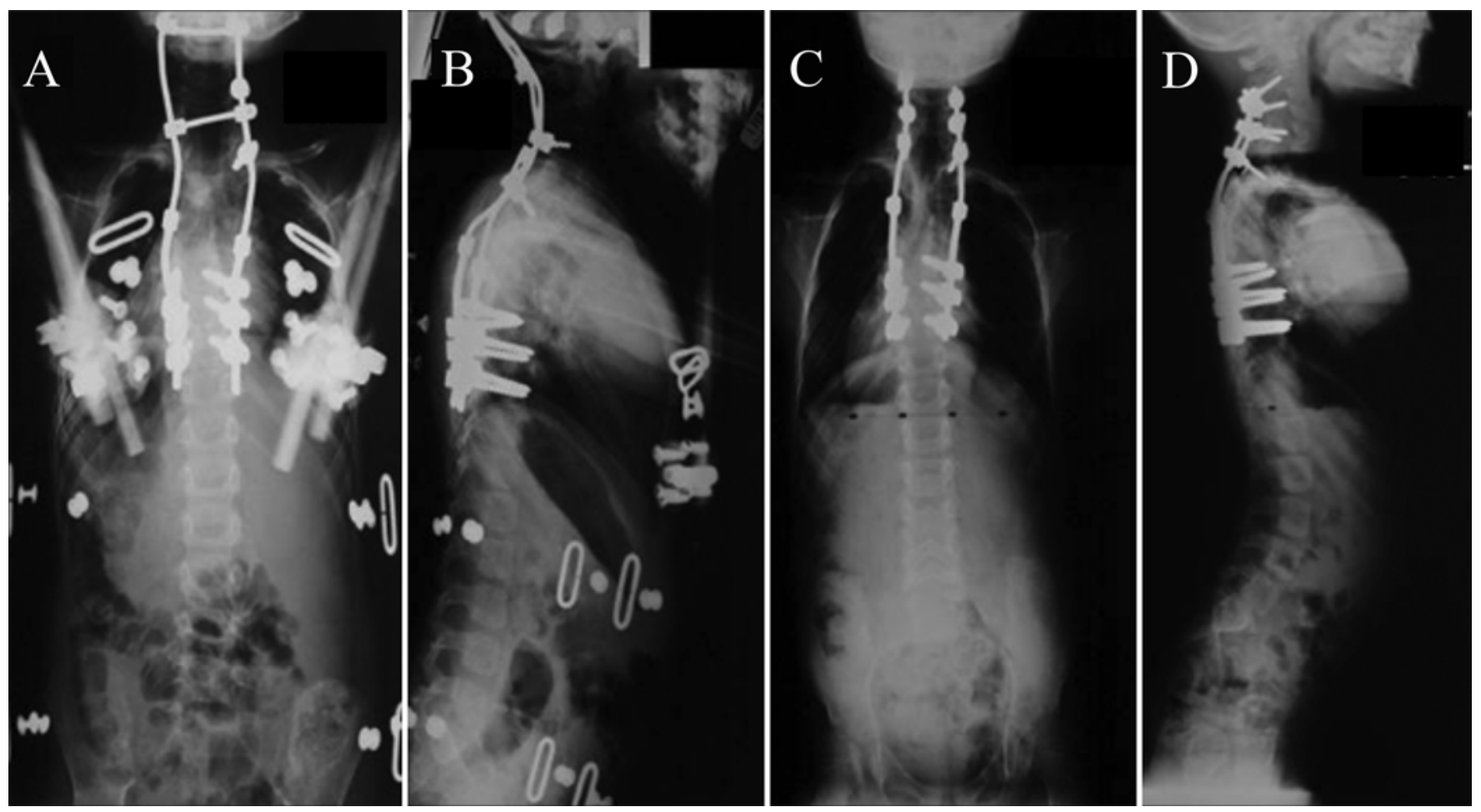

FIG. 7. Case 3. Upright AP (A) and lateral (B) radiographs after posterior instrumentation from the occiput to T-10, T-3, and T-4 vertebral column resection, and posterior fusion excluding the CVJ. Upright AP (C) and lateral (D) radiographs obtained 1 year later, after disconnection of the occipital instrumentation.

cervical spine surgical options such as anterior interbody fusion or posterior lateral mass or pedicle screw placement. When appropriate, surgical intervention is often deferred as long as possible, with children treated nonoperatively until additional spinal growth and skeletal maturity can be achieved. In some cases, however, nonoperative treatment fails and progressive neurological decline mandates surgical intervention.

Although numerous studies have described and validated occipital-cervical instrumentation and fusion as a viable treatment option for instability at the $\mathrm{CVJ},{ }^{1,5}$ there have been very few reports discussing treatment options for severe cervical or cervical-thoracic deformity in very young children. Fargen and colleagues, for example, reported a series of children who underwent complete instrumentation and fusion from the occiput to the upper thoracic spine. ${ }^{3}$ Occipital-cervical-thoracic stabilization is typically reserved for patients with severe deformity and neurological compromise, but loss of cervical spine growth and motion are significant disadvantages of this procedure.

The concept of temporary screw fixation in spinal deformity surgery is not novel. For example, extension of instrumented constructs to the pelvis but without arthrodesis (e.g., iliac screws) is commonly performed to increase stability and enhance arthrodesis in the lumbosacral region. ${ }^{6,7}$ However, because pelvic screws limit mobility, they may be removed after successful fusion of the remaining construct is confirmed. Similarly, over the last 20 years, several growth-friendly surgical strategies have been developed for the thoracolumbar spine, to control spinal deformity while preserving spinal growth. These include traditional growing rod constructs, VEPTR, and magnetically manipulated growing rods (i.e., MAGEC). ${ }^{9}$ These devices can often control and improve spinal deformity while preserving some growth potential. Unfortunately, similar devices and techniques have not been developed for the growing pediatric cervical spine. This is probably because of the anatomical constraints of the cervical spine and the relatively infrequent occurrence of complex pediatric cervical spinal deformities requiring surgical intervention at such a young age. Although our technique should not be equated to so-called growth-friendly thoracolumbar systems, temporary rather than permanent occipital fixation is an initial attempt to maintain some growth and motion at the CVJ.

\section{Conclusions}

Although limited in its scope, to our knowledge this is the first report to describe a surgical option that temporarily fixes instrumentation to the occiput to achieve arthrodesis within the cervical or cervical-thoracic spine, while also allowing for subsequent instrumentation removal and restoration of potential growth and motion at the CVJ. Formal assessment of the degree of motion after removal of occipital instrumentation was not performed due to the small number of patients in this study and because baseline motion studies (which may be limited due to underlying genetic conditions; e.g., VATER) were not available. We acknowledge that the relatively short-term follow-up of these patients does not allow for any conclusions regarding long-term outcomes of related comorbidities such 
as potential pulmonary and cardiac pathologies. It will be crucial to follow these patients through adolescence to evaluate for complications related to cervical and cervicalthoracic fusion prior to skeletal maturity.

\section{References}

1. Anderson RC, Ragel BT, Mocco J, Bohman LE, Brockmeyer DL: Selection of a rigid internal fixation construct for stabilization at the craniovertebral junction in pediatric patients. $\mathbf{J}$ Neurosurg 107 (1 Suppl):36-42, 2007

2. Campbell RM Jr, Smith MD, Mayes TC, Mangos JA, WilleyCourand DB, Kose N, et al: The characteristics of thoracic insufficiency syndrome associated with fused ribs and congenital scoliosis. J Bone Joint Surg Am 85-A:399-408, 2003

3. Fargen KM, Anderson RC, Harter DH, Angevine PD, Coon VC, Brockmeyer DL, et al: Occipitocervicothoracic stabilization in pediatric patients. J Neurosurg Pediatr 8:57-62, 2011

4. Haher TR, Yeung AW, Caruso SA, Merola AA, Shin T, Zipnick RI, et al: Occipital screw pullout strength. A biomechanical investigation of occipital morphology. Spine (Phila Pa 1976) 24:5-9, 1999

5. Hedequist DJ, Emans JB: Cervical spine instrumentation in children. J Am Acad Orthop Surg 24:370-378, 2016

6. Hyun SJ, Rhim SC, Kim YJ, Kim YB: A mid-term follow-up result of spinopelvic fixation using iliac screws for lumbosacral fusion. J Korean Neurosurg Soc 48:347-353, 2010

7. Kato S, Lewis SJ: Temporary iliac fixation to salvage an acute L4 chance fracture following pedicle screw fixation for adolescent idiopathic scoliosis. Spine (Phila Pa 1976) 42:E313-E316, 2017

8. Kennedy BC, D'Amico RS, Youngerman BE, McDowell MM, Hooten KG, Couture D, et al: Long-term growth and alignment after occipitocervical and atlantoaxial fusion with rigid internal fixation in young children. J Neurosurg Pediatr 17:94-102, 2016

9. Skaggs DL, Akbarnia BA, Flynn JM, Myung KS, Sponseller PD, Vitale MG: A classification of growth friendly spine implants. J Pediatr Orthop 34:260-274, 2014

\section{Disclosures}

Dr. Lenke is a patent holder and a consultant, and receives royalties from Medtronic. He receives support for a non-study-related clinical or research effort that he oversees from EOS Technologies, Diers Technologies, Setting Scoliosis Straight Foundation, and AOSpine.

\section{Author Contributions}

Conception and design: Anderson, Kelley, Vitale, Lenke. Acquisition of data: Anderson, Kelley, Minkara. Analysis and interpretation of data: Anderson, Kelley, Angevine, Vitale, Lenke. Drafting the article: Anderson, Kelley. Critically revising the article: Anderson, Kelley, Vitale, Lenke. Reviewed submitted version of manuscript: Anderson, Kelley.

\section{Correspondence}

Richard C. E. Anderson, Neurological Institute, 710 West 168th St., New York, NY 10032. email: rca24@cumc.columbia.edu. 